Bone and Soft Tissue Tumor

\title{
Outcomes of Adult Ewing Sarcoma Treated with Multimodality Therapy: A Single-Institute Experience
}

\section{Ravi Chandran Ambalathandi ${ }^{1}$ Siva Prasad Kuruva ${ }^{1}$ Meher Lakshmi Konatam ${ }^{1}$ Sadashivudu Gundeti ${ }^{1}$ \\ ${ }^{1}$ Department of Medical Oncology, Nizam's Institute of Medical Sciences, Hyderabad, Telangana, India}

South Asian J Cancer 2021;9:191-194.

\section{Rachana Chennamaneni ${ }^{1}$ Stalin Bala}

Address for correspondence Dr. Siva Prasad Kuruva, MBBS, MD, DM, Department of Medical Oncology, Nizam's Institute of Medical Sciences, Hyderabad, Telangana, India (e-mail: ksivaprasadkg@gmail.com).

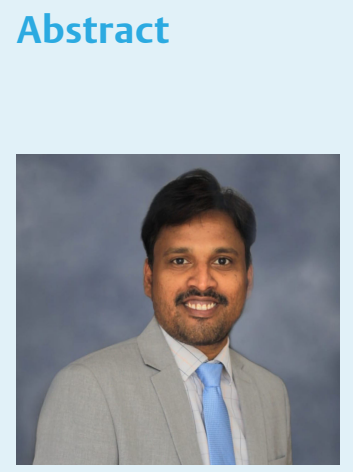

Dr. Siva Prasad Kuruva

Keywords

- adult Ewing sarcoma

- dose-dense therapy

- multimodality therapy
Introduction Ewing sarcoma (ES) is more common in children and relatively rare in adults. Adult ES has poor prognosis than children. Treatment approaches for adults have been extrapolated from pediatric experience. Data on adult ES are very few because of its rarity in adults. The present study was done to analyze the clinical profile and outcome of adult ES.

Aims The aim was to study the clinical and pathological treatment and outcomes in adult ES.

Subjects and Methods Between 2010 and 2017, a total of 73 ES patients with age more than 18 years were retrospectively analyzed. Survival analysis was done by plotting Kaplan-Meier curves.

Results A total of 73 patients were diagnosed with ES during 2010 to 2017. Among them, 43 (58.9\%) had localized disease with a median age of 24.5 years. Males were $44(60.3 \%)$ and females were 29 (39.7). Pain (75.3\%) was the most common symptom at presentation. Nine patients had incomplete details and were excluded from the analysis. Among 21 (28.8\%) patients, the lung (61.9\%) was the most common site of metastasis followed by the bone, bone marrow, and brain. The median number of chemotherapy cycles in the localized disease was 14 (range 1-17), and in metastatic disease, it was 4 (range $1-7$ ). Univariate analysis was done with respect to age ( $<25 \mathrm{vs.} \geq 25$ ), gender, elevated or normal serum lactate dehydrogenase level, tumor size $(<8 \mathrm{~cm}$ versus $\geq 8 \mathrm{~cm}$ ), site (axial versus extremity), and neoadjuvant chemotherapy (NACT) given or not. NACT had a significant impact on overall survival (OS) and the rest had no effect. At a median follow-up of 40 months, the 3 -year OS in localized disease was $87.4 \%$. In metastatic disease, the median OS was 13 months with 3 -year OS of $26 \%$.

Conclusions Outcomes with multimodality therapy in adult ES patients with localized disease are comparable to that of a pediatric cohort. However, metastatic disease has poor survival.
DOI https://doi.org/10.1055/s-0041-1723108 ISSN 2278-330X.

How to cite this article: Ambalathandi R. C, Kuruva S. P, Chennamaneni R, Bala S, Konatam M. L, Gundeti S. Outcomes of Adult Ewing Sarcoma Treated with Multimodality Therapy: A SingleInstitute Experience. South Asian J Cancer 2021;9(4):191-194. (c) 2021. MedIntel Services Pvt Ltd.

This is an open access article published by Thieme under the terms of the Creative Commons Attribution-NonDerivative-NonCommercial-License, permitting copying and reproduction so long as the original work is given appropriate credit. Contents may not be used for commercial purposes, or adapted, remixed, transformed or built upon. (https://creativecommons.org/licenses/by-nc-nd/4.0/) Thieme Medical and Scientific Publishers Pvt. Ltd., A-12, 2nd Floor, Sector 2, Noida-201301 UP, India 


\section{Introduction}

Ewing sarcoma (ES) is the second most common primary bone tumor. Approximately 70\% are diagnosed in childhood, while the rest occur in adults. ${ }^{1}$ ES is considered part of the primitive neuroectodermal family of tumors harboring characteristic translocation between chromosomes 11 and 22. The gold standard for diagnosing ES is the demonstration of $t(11$; 22) by fluorescent in situ hybridization (FISH) or polymerase chain reaction (PCR). ES occurs in both osseous and extraosseous sites with poor survival outcomes in certain sites such as the pelvis. Currently, the standard practice is multimodality management, typically with induction chemotherapy, followed by definitive local therapy with surgery and/ or radiotherapy. ${ }^{2-8}$ Historically, vincristine, doxorubicin, and cyclophosphamide (VDC) regimen was used. The introduction of ifosfamide and etoposide (IE) to the VDC regimen resulted in a significant improvement in the survival. ${ }^{4}$ Further studies showed that intensification with 2-weekly administration of VDC/IE regimen instead of conventional 3-weekly regimen has further improved survival in localized adult ES patients. ${ }^{6}$

Treatment for metastatic ES is mainly palliative in nature although a fraction of these patients may have a significantly long survival, especially those with limited metastasis in sites such as the lung. There are no specific management guidelines for adult ES. Treatment approaches for adults are based on pediatric protocols. Majority of these trials had incorporated only 9 to $15 \%$ of patients with age $>18$ years. ${ }^{2-6}$ Historically, adult ES has been considered to have a poor prognosis compared with children., ${ }^{2,-6}$ Data on adult ES from the Indian subcontinent are scant. Most of them are studies, case series, or case reports on pediatric ES. The objective of the current study is to analyze the demographic, clinicopathologic features, and survival of localized and metastatic adult ES treated with multimodality and multiagent chemotherapy.

\section{Subjects and Methods}

This is a retrospective, observational study. Data of patients aged more than 18 years with ES diagnosed between 2010 and 2017 were analyzed for demographic and clinicopathologic features and survival outcomes. The diagnosis of ES was confirmed by local part imaging with MRI, biopsy, and FISH for ES breakpoint region 1 (EWSR1). The staging evaluation included a bone marrow aspiration and biopsy, CT chest, and a bone scan.

Most of the patients with the localized disease received neoadjuvant chemotherapy (NACT), followed by definitive surgery and/or radiation and adjuvant chemotherapy (ACT) thereafter. The chemotherapy regimen VDC/IE was given for a duration of 1 year. Some patients had surgery or radiotherapy upfront, followed by ACT.

Patients with the metastatic disease received palliative chemotherapy with the VDC regimen. Palliative radiation was given to selected patients. Those patients with localized or metastatic disease who received treatment for at least one cycle of chemotherapy were analyzed for survival rates.
Patients who lost to follow-up were censored. Overall survival (OS) was defined as the time from diagnosis to the time of death due to any cause or lost to follow-up.

\section{Statistical Methods}

For demographic data, descriptive statistics were used. Tumor assessment was done using the Response Evaluation Criteria in Solid Tumors version 1.1. OS was analyzed by the Kaplan-Meier method. Univariate analysis was done to assess the effect of age, gender, primary tissue type, primary site of the disease, and size of the primary tumor on OS in localized ES. All statistical analyses were done using SPSS software for Windows version 25.0 (IBM Corp. Released 2017. Armonk, NY, USA.).

\section{Results}

\section{Baseline Characteristics}

A total of 73 patients with adult ES (age $>18$ years) were seen between 2010 and 2017, of which 43 (58.9\%) had localized disease. Data on treatment details were not available for nine patients and they were excluded from the survival analysis. The median age at diagnosis was 24.5 years (range 18-48 years). There were 44 (60.3\%) males and 29 (39.7\%) females. Pain and swelling were the common presenting complaints in $55(75.3 \%)$ and 31 (42.5\%) patients, respectively. Forty-two (57.5\%) patients had an osseous origin of the primary tumor and the rest had an extraosseous origin. Sites of involvement were extremity in 34 (46.6\%) patients, chest wall in 19 (26\%), axial skeleton in 13 (17.8\%), and seven patients had other sites. Median tumor size was $6.5 \mathrm{~cm}$ (range 2-21 cm). Elevated lactate dehydrogenase (LDH) enzyme level was seen in 19 (26\%) patients.

Of $21(28.8 \%)$ patients with metastatic disease, the lung (61.9\%) was the most common site of metastasis, followed by the bone $(6.5 \%)$, bone marrow $(4.8 \%)$, and brain (4.8\%). Four patients $(19 \%)$ had multiple sites of metastasis. The baseline demographic and pathologic parameters are shown in - Table 1.

\section{Treatment}

Among 43 localized adult ES patients, initial induction chemotherapy for 12 weeks with VDC/IE regimen was given in $26(60.5 \%)$ patients. Sixteen patients (37.2\%) had upfront definitive surgery and diagnosed as ES postoperatively. Upfront radical radiotherapy was given in one patient (2.3\%). All patients were offered ACT. The median number of chemotherapy cycles was 14 (range 1-17). The median number of chemotherapy cycles was four (range 1-7) in patients with metastatic disease and five (23.8\%) in patients who received palliative radiation.

\section{Variables Affecting Survival}

Univariate analysis was done with various clinicopathological features to determine the significance on survival. Variables tested for significance on survival are age ( $<26$ vs. $\geq 26$ years), gender (male vs. female), primary site (extremity vs. axial), tumor size $(<8 \mathrm{~cm}$ vs. $>8 \mathrm{~cm})$, serum LDH level (elevated vs. 
Table 1 Demographic and treatment details

\begin{tabular}{|c|c|}
\hline Parameter & $n$ \\
\hline \multicolumn{2}{|l|}{ Gender } \\
\hline Male & $44(60.3 \%)$ \\
\hline Female & $29(39.7 \%)$ \\
\hline \multicolumn{2}{|l|}{ Symptoms } \\
\hline Pain & $55(75.3 \%)$ \\
\hline Swelling & $31(42.5 \%)$ \\
\hline Constitutional symptoms & $14(19.2 \%)$ \\
\hline \multicolumn{2}{|l|}{ Primary tissue type } \\
\hline Osseous & $42(57.5)$ \\
\hline Extraosseous & $31(42.5)$ \\
\hline \multicolumn{2}{|l|}{ Primary site of disease } \\
\hline Extremity & $34(46.6 \%)$ \\
\hline Chest wall & $19(26.0 \%)$ \\
\hline Axial skeleton & $13(17.8 \%)$ \\
\hline Other sites & $07(09.6 \%)$ \\
\hline \multicolumn{2}{|l|}{ Tumour Size $(\mathrm{cm})$} \\
\hline$<8$ & $47(64.4 \%)$ \\
\hline$>8$ & $26(35.6 \%)$ \\
\hline \multicolumn{2}{|l|}{ Metastatic disease } \\
\hline Absent & $43(58.9 \%)$ \\
\hline Present & $21(28.8 \%)$ \\
\hline No data & $09(12.3 \%)$ \\
\hline \multicolumn{2}{|l|}{ Sites of metastasis } \\
\hline Lung & $13(61.9 \%)$ \\
\hline Bone & $02(09.5 \%)$ \\
\hline Bone marrow & $01(04.8 \%)$ \\
\hline Brain & $01(04.8 \%)$ \\
\hline Multiple sites & $04(19.0 \%)$ \\
\hline \multicolumn{2}{|l|}{ Treatment sequence (localized disease) } \\
\hline Initial induction chemotherapy & $26(60.5 \%)$ \\
\hline \multicolumn{2}{|l|}{ Initial definitive therapy } \\
\hline Surgery & $16(37.2 \%)$ \\
\hline Radiation & $01(02.3 \%)$ \\
\hline \multicolumn{2}{|l|}{$\begin{array}{l}\text { Treatment in metastatic disease } \\
\text { (minimum of } 4 \text { cycles) }\end{array}$} \\
\hline Treatment received & $10(55.6 \%)$ \\
\hline No treatment & $08(44.4 \%)$ \\
\hline Parameter & Range (Median) \\
\hline Age in years & $18-58(24.5)$ \\
\hline LDH U/L & $140-1913(405.5)$ \\
\hline $\begin{array}{l}\text { No. of chemotherapy cycles in localized } \\
\text { ES }\end{array}$ & $1-19(14)$ \\
\hline $\begin{array}{l}\text { No. of chemotherapy cycles in meta- } \\
\text { static ES }\end{array}$ & $1-6(04)$ \\
\hline
\end{tabular}

Abbreviations: ES-Ewing Sarcoma; LDH-Lactate dehydrogenase. normal), and NACT (yes vs. no). On univariate analysis, NACT had a significant impact ( $p=0.35$ ) on OS, while age, gender, LDH, primary site, and tumor size had no impact.

\section{Survival}

At a median follow-up of 40 months, the 3 -year OS in patients with localized disease was $87.4 \%$. At a median follow-up of 13 months, the median OS in patients with metastatic disease was 13 months with a 3 -year survival of $26 \%$. KaplanMeier survival curves are shown in -Fig. 1 .

\section{Discussion}

This is perhaps the first study from the Indian subcontinent on adult ES using the VAD/IE chemotherapy protocol. Similar to acute lymphoblastic leukemia, the treatment protocols for the adult ES were inspired from pediatric ES studies. The inclusion of adults in pediatric trials was limited. Even though these landmark studies included patients aged $>18$ years, they accounted for 9 to $15 \%$ only. ${ }^{2-6}$

In the current study, the median age at the time of diagnosis was 24.5 years and males accounted for $60.3 \%$. The largest series of ES was studied by Verma et al $(n=1870)$, who reported the median age as 29 years. ${ }^{9}$ Other studies by Ahmed et al, Pretz et al, and Verrill et al reported a median age of 27.5, 28 , and 24 years, respectively. ${ }^{10-12}$ In this study, there was a male predominance $(60.3 \%)$, similar to that of the studies by Verma et al (60\%), Ahmed et al (67.7\%), and Verrill et al (62.7\%). Pretz et al had a female predominance, accounting for $60 \%$.

In this study, pain and swelling were the most common presenting complaints seen in $75.3 \%$ and $42.5 \%$ patients, respectively, which is similar to other studies. The extremity was the most common site in majority of studies except in the study by Verma et al where the axial skeleton was most commonly involved.

Preoperative chemotherapy was given to $60.5 \%$ of the patients and $37.2 \%$ of the patients had undergone upfront curative surgery, who were diagnosed with ES

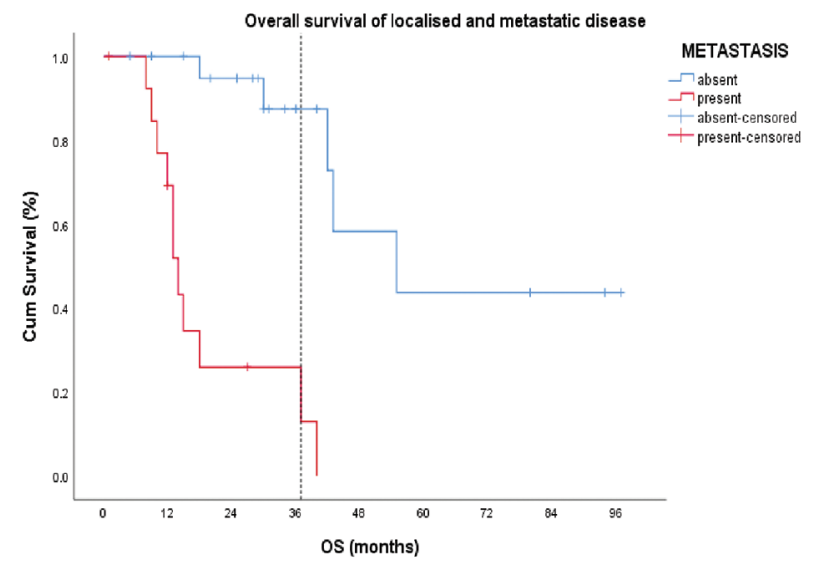

Fig. 1 Overall survival of localized and metastatic disease by KaplanMeier estimates. 
on pathological examination of the tumor. One patient received local radical radiotherapy as therapy. Most of the patients received preoperative chemotherapy, followed by local control with either surgery and/or radiotherapy. Our data reflected the real-world practice of localized ES patients.

For patients with localized disease who received multimodality therapy, the 3 -year OS was $87.4 \%$. According to the historical data, before the introduction of VDC/IE regimen, the 5 -year OS was 20 to $60 \%$. This increased to more than $80 \%$ with the adoption of the VDC/IE regimen. Ahmed et al studied this impact of the addition of therapy in their recent study, showing improvement of 5-year OS from $49 \%$ to $73 \%$, done between 1977 to 1992 and 1993 to 2007 , respectively.$^{10} \mathrm{~A}$ similar study by Nasaka et al reported the impact of the chemotherapy regimen on ES. ${ }^{13}$ She reported increased OS with the VDC/IE regimen. A recent study on adult ES by Pretz et al showed a 5-year OS of $79 \%$.

In this study, $28.8 \%$ of the patients were diagnosed with a metastatic disease, which is similar to the study reported by Verma et al (33\%) and Verrill et al (28.8\%). Pretz and Varma's studies reported lung as the most common site of metastasis, as was found in this study. However, Verrill et al reported bone as the frequent metastatic site. All patients in this study were given VDC-based regimen and $23.5 \%$ required radiation for palliation of symptoms. For metastatic ES, the 3 -year OS was $13 \%$, while it was $10 \%$ and $0 \%$ in studies by Verma et al and Verrill et al, respectively.

Retrospective nature, limited sample size, and missing data in a few patients are the limitations of this study. However, these problems reflect the ground reality of real-world scenario while treating patients with adult ES.

\section{Conclusions}

With the introduction of VDC/IE regimen, survival has dramatically improved. Metastatic ES patients still have adverse outcomes, pressing the need for additional therapies in future. Adoption of dose-dense regimens in select patients may further increase the survival.

\section{Financial Support and Sponsorship \\ Nil.}

\section{Conflicts of Interest}

None declared.

\section{Acknowledgment}

We sincerely thank all the faculty of the Pathology and Orthopedic department, Nizam's Institute of Medical Sciences, Hyderabad.

\section{References}

1 Glass AG, Fraumeni JF Jr. Epidemiology of bone cancer in children. J Natl Cancer Inst 1970;44(1):187-199

2 Nesbit ME Jr, Gehan EA, Burgert EOJr, et al. Multimodal therapy for the management of primary, nonmetastatic Ewing's sarcoma of bone: a long-term follow-up of the First Intergroup study. J Clin Oncol 1990;8(10):1664-1674

3 Burgert EO Jr, Nesbit ME, Garnsey LA, et al. Multimodal therapy for the management of nonpelvic, localized Ewing's sarcoma of bone: intergroup study IESS-II. J Clin Oncol 1990;8(9):1514-1524

4 Grier HE, Krailo MD, Tarbell NJ, et al. Addition of ifosfamide and etoposide to standard chemotherapy for Ewing's sarcoma and primitive neuroectodermal tumor of bone. $\mathrm{N}$ Engl J Med 2003;348(8):694-701

5 Granowetter L, Womer R, Devidas M, et al. Dose-intensified compared with standard chemotherapy for nonmetastatic Ewing sarcoma family of tumors: a Children's Oncology Group Study. J Clin Oncol 2009;27(15):2536-2541

6 Womer RB, West DC, Krailo MD, et al. Randomized controlled trial of interval-compressed chemotherapy for the treatment of localized Ewing sarcoma: a report from the Children's Oncology Group. J Clin Oncol 2012;30(33):4148-4154

7 Lin PP, Jaffe N, Herzog CE, et al. Chemotherapy response is an important predictor of local recurrence in Ewing sarcoma. Cancer 2007;109(3):603-611

8 Gaspar N, Hawkins DS, Dirksen U, et al. Ewing sarcoma: current management and future approaches through collaboration. J Clin Oncol 2015;33(27):3036-3046

9 Verma V, Denniston KA, Lin CJ, Lin C. A comparison of pediatric versus adult patients with the Ewing sarcoma family of tumors. Front Oncol 2017;7:82

10 Ahmed SK, Robinson SI, Okuno SH, Rose PS, Laack NN. Adult ewing sarcoma: survival and local control outcomes in 102 patients with localized disease. Sarcoma 2013;2013:681425

11 Pretz JL, Barysauskas CM, George S, et al. Localized adult Ewing sarcoma: favorable outcomes with alternating vincristine, doxorubicin, cyclophosphamide, and ifosfamide, etoposide (VDC/IE)-based multimodality therapy. Oncologist 2017;22(10):1265-1270

12 Verrill MW, Judson IR, Wiltshaw E, Thomas JM, Harmer CL, Fisher C. The use of paediatric chemotherapy protocols at full dose is both a rational and feasible treatment strategy in adults with Ewing's family tumours. Ann Oncol 1997;8(11):1099-1105

13 Nasaka S, Gundeti S, Ganta RR, Arigela RS, Linga VG, Maddali LS. Impact of treatment protocol on outcome of localized Ewing's sarcoma. South Asian J Cancer 2016;5(4):194195Kumar Prabhash, DM 